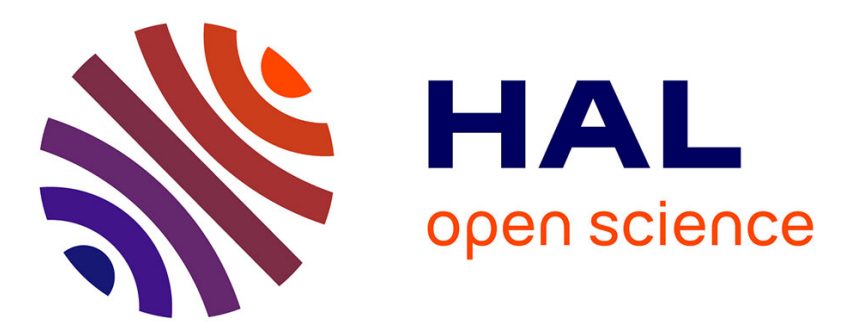

\title{
Inference with a contrast-based posterior distribution and application in spatial statistics
}

Samuel Soubeyrand, Florence Carpentier, Nicolas Desassis, Joël Chadœuf

\section{To cite this version:}

Samuel Soubeyrand, Florence Carpentier, Nicolas Desassis, Joël Chadœuf. Inference with a contrastbased posterior distribution and application in spatial statistics. Statistical Methodology, 2009, 6 (5), pp.466-477. 10.1016/j.stamet.2009.03.003 . hal-02409013

\section{HAL Id: hal-02409013 https://hal.science/hal-02409013}

Submitted on 30 May 2020

HAL is a multi-disciplinary open access archive for the deposit and dissemination of scientific research documents, whether they are published or not. The documents may come from teaching and research institutions in France or abroad, or from public or private research centers.
L'archive ouverte pluridisciplinaire HAL, est destinée au dépôt et à la diffusion de documents scientifiques de niveau recherche, publiés ou non, émanant des établissements d'enseignement et de recherche français ou étrangers, des laboratoires publics ou privés. 


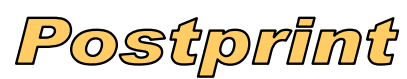

Version définitive du manuscrit publié dans / Final version of the manuscript

published in : Statistical Methodology, 2009, DOI: 10.1016/j.stamet.2009.03.003

\title{
Inference with a contrast-based posterior distribution and application in spatial statistics
}

\author{
S. Soubeyrand ${ }^{* \dagger}$, F. Carpentier*, N. Desassis ${ }^{\ddagger}$ and J. Chadœuf* \\ May 14, 2009
}

\begin{abstract}
The likelihood function is often used for parameter estimation. Its use, however, may cause difficulties in specific situations. In order to circumvent these difficulties, we propose a parameter estimation method based on the replacement of the likelihood in the formula of the Bayesian posterior distribution by a function which depends on a contrast measuring the discrepancy between observed data and a parametric model. The properties of the contrast-based (CB) posterior distribution are studied to understand what the consequences of incorporating a contrast in the Bayes formula are. We show that the $\mathrm{CB}-$ posterior distribution can be used to make frequentist inference and to assess the asymptotic variance matrix of the estimator with limited analytical calculations compared to the classical contrast approach. Even if the primary focus of this paper is on frequentist estimation, it is shown that for specific contrasts the CB-posterior distribution can be used to make inference in the Bayesian way.

The method was used to estimate the parameters of a variogram (simulated data), a Markovian model (simulated data) and a cylinder-based autosimilar model describing soil roughness (real data). Even if the method is presented in the spatial statistics perspective, it can be applied to non-spatial data.
\end{abstract}

\footnotetext{
${ }^{*}$ INRA, UR546 Biostatistique et Processus Spatiaux, F-84914 Avignon, France

${ }^{\dagger}$ Corresponding author: samuel.soubeyrand@avignon.inra.fr

${ }^{\ddagger}$ Ecole Nationale Supérieure des Mines de Paris, Centre de Géosciences, F-77300 Fontainebleau, France
} 


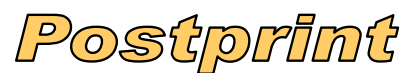

Version définitive du manuscrit publié dans / Final version of the manuscript

published in : Statistical Methodology, 2009, DOI: 10.1016/j.stamet.2009.03.003

Key words. Frequentist estimation; Quasi-Bayesian estimation; Spatial model.

\section{Introduction}

In both the frequentist and the Bayesian viewpoints, the likelihood function has become the major component of statistical inference under a parametric model. Its use, however, has drawbacks in specific situations. First, it may be impossible to write down the likelihood in a numerically tractable form; see the cases of Boolean models (Van Lieshout and Van Zwet, 2001), Markov point processes (Møller, 2003), Markov spatial processes (Guyon, 1985) and spatial generalized linear mixed models (spatial GLMM; Diggle et al., 1998) where multiple integrals cannot be reduced due to spatial dependences. Second, the likelihood may not be completely appropriate because of the associated assumptions. For instance, the likelihood is built under an assumption on the distribution of data, but such an assumption may be tricky to specify in the case of insufficient information as in classical geostatistics (Chilès and Delfiner, 1999); see also McCullagh and Nelder (1989, chap. 9). In the same vein, every data are assumed to have the same weights in the likelihood, but the influence of outliers may be too large according to the analyst (Markatou, 2000).

The difficulties encountered with the likelihood can be circumvented with the existing Bayesian and frequentist procedures.

- There are procedures which use conditional simulations to numerically approximate the likelihood. For instance, Markov chain Monte Carlo algorithms (MCMC; Robert and Casella, 1999) allow the approximation of the posterior distribution for Markov point processes (Møller, 2003) and spatial GLMMs (Diggle et al., 1998); Markov chain expectation maximization algorithms (MCEM; Wei and Tanner, 1990) allow the maximization of the likelihood for Boolean models (Van Lieshout and Van Zwet, 2001) and spatial GLMMs (Zhang, 2002).

- There are procedures where the likelihood function is simplified or replaced. For example, the likelihood can be replaced by a pseudo-likelihood which 


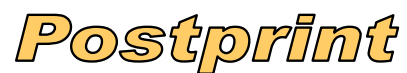

Version définitive du manuscrit publié dans / Final version of the manuscript

published in : Statistical Methodology, 2009, DOI: 10.1016/j.stamet.2009.03.003

only takes account of local dependence structures, like in Møller (2003) for Markov point processes and Besag (1975) and Guyon (1985) for Markov spatial processes. The generalized least squares estimation, which does not rely on assumptions on the distribution of data, is used in geostatistics for the estimation of variogram parameters; see Chilès and Delfiner (1999, chap. 2-3) and Stein (1999, chap. 1). Other examples of procedures where the likelihood function was replaced are: the weighted likelihood maximization (Markatou, 2000), the method of moments, the M-estimation (Serfling, 2002), the approximate Bayesian computation (ABC; Beaumont et al., 2002), the pseudo-posterior approach of Walker and Hjort (2001), the quasi-likelihood maximization (McCullagh and Nelder, 1989) and the quasi-Bayesian likelihood method (Lin, 2006).

In the pseudo-posterior approach of Walker and Hjort (2001), the likelihood is replaced by its square-root in the posterior distribution. This has the consequence of flattening the likelihood and leads to a robustifying posterior distribution. In the quasi-Bayesian likelihood approach (Lin, 2006), the likelihood appearing in the posterior distribution formula is replaced by a quasi-likelihood which does not rely on distribution assumptions. Then, the posterior distribution which is obtained is used to make inference as in classical Bayesian situations. In this communication we propose to generalize these approaches: the likelihood in the posterior distribution formula is replaced by a function of a contrast.

A contrast is a function of the model parameters and the observed data which is minimized to estimate the parameters (Dacunha-Castelle and Duflo, 1982). The minimum contrast approach is a generic estimation method which was developed in a frequentist perspective. The maximum likelihood estimation as well as the maximum pseudo, weighted or quasi likelihood estimation, the diverse least squares methods, the method of moments and the M-estimation can be formulated as minimum contrast estimation problems.

The procedure which is proposed - replacing the likelihood by a function of a contrast in the Bayesian formula - generalizes some of the existing approaches. It indeed includes the classical Bayesian approach (here and thereafter "classical" refers to "likelihood-based"), the pseudo-posterior approach of Walker and Hjort 
(2001) and the quasi-Bayesian approach of Lin (2006). This procedure provides a contrast-based $(\mathrm{CB})$ posterior distribution which does not coincide, in the general case, with the classical posterior distribution. In this paper, we investigate what are the posterior distribution and the MAP (maximum a posteriori) estimator based on a contrast.

Under mild conditions on the prior distribution, we show that the CB-MAP estimator inherits the asymptotic properties (consistency and asymptotic normality) of the minimum contrast estimator, as the classical MAP estimator inherits the asymptotic properties of the maximum likelihood estimator (Caillot and Martin, 1972). The limit variance matrix of the normalized estimator is $I_{\theta}^{-1} \Gamma_{\theta} I_{\theta}^{-1}$ where $\Gamma_{\theta}$ is the limit variance of the gradient of the contrast and $I_{\theta}$ is the limit Hessian matrix of the contrast.

Moreover, we show that the $\mathrm{CB}$-posterior distribution is asymptotically equivalent to a normal distribution whose variance matrix is $I_{\theta}^{-1}$. Therefore, when building the contrast, particular attention must be paid to satisfy, if possible, $I_{\theta}^{-1} \Gamma_{\theta} I_{\theta}^{-1}=I_{\theta}^{-1}$. Indeed, with such a contrast, inference can be made without computing matrices $\Gamma_{\theta}$ and $I_{\theta}$ : the posterior distribution can either be used as a limit distribution in a frequentist viewpoint or be used to make inference in the Bayesian way. When building a contrast satisfying $I_{\theta}^{-1} \Gamma_{\theta} I_{\theta}^{-1}=I_{\theta}^{-1}$ is not possible, the CB-posterior distribution can nevertheless be used to estimate $I_{\theta}^{-1}$. Thus, the computation of the limit Hessian matrix of the contrast is avoided.

The article is organized as follows. The method is presented in section 2, and its properties are derived. Then, it is applied in sections 3 and 4 to simulated and real cases in spatial statistics (estimation of the range parameter of a variogram; estimation of the parameters of a Markovian spatial process; and estimation of the parameters of a cylinder-based autosimilar model describing soil roughness). The three cases illustrate the application of the method when the parameter has one or several components and when $I_{\theta}^{-1} \Gamma_{\theta} I_{\theta}^{-1}$ is equal to or different from $I_{\theta}^{-1}$. 


\section{Incorporating a contrast in the Bayesian for- mula}

\subsection{Minimum contrast estimation}

Consider a family of parametric models $\left\{P_{\alpha}: \alpha \in \Theta\right\}$ and samples of increasing sizes $t \in T \subset \mathbb{N}$, drawn from the model $P_{\theta}$ with the true parameter $\theta$. A contrast for $\theta$ is a random function $\alpha \mapsto U_{t}(\alpha)$ defined over $\Theta$, depending on a sample of size $t$, and such that $\left\{U_{t}(\alpha): t \in T\right\}$ converges in probability, as $t \rightarrow \infty$, to a function $\alpha \mapsto K(\alpha, \theta)$ which has a strict minimum at $\alpha=\theta$. The minimum contrast estimator is

$$
\hat{\theta}_{t}=\operatorname{argmin}\left\{U_{t}(\alpha), \alpha \in \Theta\right\} .
$$

The maximum likelihood estimation, the maximum pseudo, weighted or quasi likelihood estimation, the diverse least squares methods, the method of moments and the M-estimation can be formulated as minimum contrast estimation problems. A detailed presentation of minimum contrast estimation can be found in Dacunha-Castelle and Duflo (1982). Molchanov (1997) illustrates the use of minimum contrast estimation in spatial statistics.

\subsection{Posterior distribution and MAP estimator based on a contrast}

Let $\left(X_{i}\right)_{i \leq t}$ be a sample of size $t$ with distribution $P_{\alpha}$. Then, the posterior distribution of $\alpha$ is

$$
p\left(\alpha \mid X_{i}, i \leq t\right)=\frac{P_{\alpha}\left(X_{i}, i \leq t\right) c(\alpha)}{\int_{\Theta} P_{\beta}\left(X_{i}, i \leq t\right) c(\beta) d \beta},
$$

where $P_{\alpha}\left(X_{i}, i \leq t\right)$ denotes the likelihood and $c(\cdot)$ is a prior distribution defined over $\Theta$. The contrast corresponding to the likelihood being $U_{t}^{l i k}(\alpha)=$ $-\frac{1}{t} \log P_{\alpha}\left(X_{i}, i \leq t\right)$ (Dacunha-Castelle and Duflo, 1982), the posterior distribution can be written by replacing $P_{\alpha}\left(X_{i}, i \leq t\right)$ by $\exp \left(-t U_{t}^{l i k}(\alpha)\right)$ in the previous equation. 
Here, we propose to substitute the contrast associated with the likelihood in the Bayesian formula with any contrast $U_{t}(\alpha)$. This leads to a contrast-based (CB) posterior distribution denoted by $p_{t}(\alpha)$ :

$$
p_{t}(\alpha)=\frac{\exp \left(-t U_{t}(\alpha)\right) c(\alpha)}{\int_{\Theta} \exp \left(-t U_{t}(\beta)\right) c(\beta) d \beta} .
$$

The CB-MAP estimator obtained by maximizing $p_{t}(\cdot)$ is denoted by

$$
\tilde{\theta}_{t}=\operatorname{argmax}\left\{p_{t}(\alpha), \alpha \in \Theta\right\}
$$

$\tilde{\theta}_{t}$ is at the minimum of $\alpha \mapsto U_{t}(\alpha)-(1 / t) \log c(\alpha)$ and, in general, does not coincide with the classical minimum contrast estimator $\hat{\theta}_{t}=\operatorname{argmin}\left\{U_{t}(\alpha), \alpha \in\right.$ $\Theta\}$.

Remark: The CB-posterior distribution proposed in equation (1) is one out of several possible alternatives to the classical posterior distribution. Indeed, other link functions, say $g$, could be used instead of the exponential function. The link $g$ should be an increasing function and should satisfy regularity conditions in order to inherit the properties of the contrast. The use of the exponential link has two major advantages: (i) the classical Bayesian approach is included in the method which is proposed and (ii) the exponential link is particularly convenient for mathematical derivations allowing us to determine the properties of the $\mathrm{CB}-\mathrm{MAP}$ estimator and the $\mathrm{CB}$-posterior distribution. These properties are investigated below.

\subsection{Consistency and asymptotic normality of the CB- MAP estimator}

We noted above that the CB-MAP estimator $\tilde{\theta}_{t}$ is at the minimum of $\alpha \mapsto$ $U_{t}(\alpha)-(1 / t) \log c(\alpha)$. This function satisfies the definition of a contrast. Consequently, convergence properties of $\tilde{\theta}_{t}$ can be easily obtained by using the contrast theory (Dacunha-Castelle and Duflo, 1982). Assume that the hypotheses required for the convergence of the classical minimum contrast estimator (see Appendix A) are satisfied. Let us assume in addition that the prior distribution $c(\cdot)$ is proper, differentiable and strictly positive over $\Theta$. It follows that, as $t \rightarrow \infty$, 


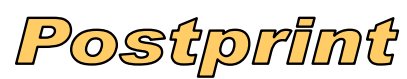

Version définitive du manuscrit publié dans / Final version of the manuscript

published in : Statistical Methodology, 2009, DOI: 10.1016/j.stamet.2009.03.003

- $\tilde{\theta}_{t}$ converges in probability to $\theta$ and

- $\sqrt{t}\left(\tilde{\theta}_{t}-\theta\right)$ converges in law to the Gaussian distribution $\mathcal{N}\left(0, I_{\theta}^{-1} \Gamma_{\theta} I_{\theta}^{-1}\right)$,

where $I_{\theta}$ and $\Gamma_{\theta}$ are matrices satisfying:

$$
\begin{aligned}
\mathbf{H} U_{t}(\theta) & \rightarrow I_{\theta} \quad \text { in probability as } t \rightarrow \infty \\
\sqrt{t} \operatorname{grad} U_{t}(\theta) & \rightarrow \mathcal{N}\left(0, \Gamma_{\theta}\right) \quad \text { in law. }
\end{aligned}
$$

Remark 1: The convergence results given above can also be obtained by noting that the asymptotic deviation between the classical minimum contrast estimator $\hat{\theta}_{t}$ and the CB-MAP estimator $\tilde{\theta}_{t}$ is of order $1 / t$. More exactly, it is shown in Appendix B that

$$
\tilde{\theta}_{t}-\hat{\theta}_{t}=\frac{1+o_{\text {proba }}(1)}{t c(\theta)} I_{\theta}^{-1} \operatorname{grad} c(\theta) .
$$

Remark 2: It would be interesting to study not only the asymptotics of the CB-posterior maximum but also the asymptotics of the CB-posterior mean and median which are commonly used in classical Bayesian estimation.

\subsection{Convergence of the $\mathrm{CB}$-posterior distribution}

The CB-posterior distribution $p_{t}(\cdot)$ is asymptotically equivalent to the density function of the Gaussian distribution $\mathcal{N}\left(\tilde{\theta}_{t},\left(t I_{\theta}\right)^{-1}\right)$ (see Appendix C):

$$
p_{t}(\alpha) \underset{t \rightarrow \infty}{\sim} \frac{1}{(2 \pi)^{p / 2}\left|\left(t I_{\theta}\right)^{-1}\right|^{1 / 2}} \exp \left(-\frac{1}{2}\left(\alpha-\tilde{\theta}_{t}\right)^{\prime}\left(t I_{\theta}\right)\left(\alpha-\tilde{\theta}_{t}\right)\right) .
$$

This result allows us to figure out what the $\mathrm{CB}$-posterior distribution is and how it can be used to make inference in the frequentist and Bayesian ways.

In the contrast theory, the distribution $\mathcal{N}\left(\tilde{\theta}_{t},\left(t I_{\theta}\right)^{-1} \Gamma_{\theta} I_{\theta}^{-1}\right)$ is used to make frequentist inference about $\theta$ : the point estimator is $\tilde{\theta}_{t}$, and confidence zones are provided based on the this normal distribution. Consequently, if the contrast is such that $I_{\theta}^{-1} \Gamma_{\theta} I_{\theta}^{-1}=I_{\theta}^{-1}$, then the $\mathrm{CB}$-posterior distribution $p_{t}(\cdot)$ which approximates the density of $\mathcal{N}\left(\tilde{\theta}_{t},\left(t I_{\theta}\right)^{-1}\right)$ can be directly used to make frequentist inference about $\theta$ : the mode of $p_{t}(\cdot)$ is the point estimator, and confidence zones can be directly determined from $p_{t}(\cdot)$. This case is particularly 
interesting since the calculation of the limit matrices $I_{\theta}=\lim _{t \rightarrow \infty} \mathbf{H} U_{t}(\theta)$ and $\Gamma_{\theta}=\lim _{t \rightarrow \infty} V_{\theta}\left(\sqrt{t} \operatorname{grad} U_{t}(\theta)\right)$ is no more required.

Moreover, when the contrast which is considered satisfies $I_{\theta}^{-1} \Gamma_{\theta} I_{\theta}^{-1}=I_{\theta}^{-1}$, we propose to use the $\mathrm{CB}$-posterior distribution $p_{t}(\cdot)$ to make inference in the Bayesian way, i.e. to use $p_{t}(\cdot)$ as a real posterior density. The motivation is based on the following analogy: when the contrast corresponding to the likelihood is employed (in this case, $I_{\theta}^{-1} \Gamma_{\theta} I_{\theta}^{-1}=I_{\theta}^{-1}$ ), then $p_{t}(\cdot)$ can be used (i) to make frequentist inference since it is an approximation of the limit distribution of the estimator (see above) and (ii) to make Bayesian inference since it is the classical posterior density. It has to be noted that, in general, the CB-posterior density $p_{t}(\cdot)$ does not coincide with the classical posterior density. It is a posterior density based on the information brought by the contrast under consideration.

If the contrast does not satisfy $I_{\theta}^{-1} \Gamma_{\theta} I_{\theta}^{-1}=I_{\theta}^{-1}$, then the CB-posterior distribution $p_{t}(\cdot)$ cannot be used to approximate the limit distribution of $\tilde{\theta}_{t}$ or to make Bayesian inference. However, $p_{t}(\cdot)$ can be used to estimate the matrix $I_{\theta}$, so avoiding the calculation of the second derivatives of the contrast. Indeed, one can see from (3) that an estimate of $I_{\theta}$ is the matrix $\Omega^{-1} / t$ where $\Omega$ is the variance matrix of the normal density function centered around $\tilde{\theta}_{t}$ and fitted to $p_{t}(\cdot)$ (using a least square technique for example). If $\theta$ is real, $I_{\theta}$ can be more simply estimated by $2 \pi p_{t}\left(\tilde{\theta}_{t}\right)^{2} / t$ since equation (3) yields $p_{t}\left(\tilde{\theta}_{t}\right) \underset{t \rightarrow \infty}{\sim}\left(t I_{\theta} / 2 \pi\right)^{1 / 2}$. We have not found an equivalent way to easily estimate $\Gamma_{\theta}$ without analytical calculation of the second derivatives and without simulations.

\section{Application in spatial statistics: simulated data}

This section illustrates the properties of the CB-posterior distribution and shows the possible discrepancy between this posterior and the limit distribution of the CB-MAP.

\subsection{Least-square estimation of a variogram range}

This simulated case illustrates the application of the method for a real parameter. Here, the $\mathrm{CB}$-posterior distribution cannot be directly used to make 
inference but can be used to estimate $I_{\theta}$.

We built a data set by simulating a centered Gaussian random field whose variogram at distance $h$ is $\gamma_{\theta}(h)=1-\exp (-\theta h)$ with $\theta=1 ; \theta$ is the inverse of the range parameter. The field was simulated over a square grid $\left\{i=\left(i_{1}, i_{2}\right)\right.$ : $\left.0 \leq i_{1}, i_{2} \leq n\right\}$ with size $t=n^{2}=20^{2}$ and inter-node distance one. Figure 1 (left) shows the simulated random field. The sample variogram $\hat{\gamma}(h)$ (Chilès and Delfiner, 1999) was estimated for every possible inter-points distance $h$ less than the half diagonal of the grid; let $\mathcal{H}$ denote the set of these distances.

For the estimation of $\theta$, we chose a uniform prior density over $[0,4]$ (horizontal dotted line in Fig. 1, right) and we used the least-square contrast between the sample variogram and the theoretical variogram

$$
U_{n^{2}}(\alpha)=\frac{1}{2} \sum_{h \in \mathcal{H}}\left\{\hat{\gamma}(h)-\gamma_{\alpha}(h)\right\}^{2} .
$$

The CB-posterior density is shown in Figure 1 (right, dotted curve). The MAP estimate is $\tilde{\theta}_{t}=1.34$ (vertical line).

Estimation uncertainty was assessed by estimating the limit variance $\Gamma_{\theta} /\left(n I_{\theta}\right)^{2}$ of $\tilde{\theta}_{t}$. The term $\Gamma_{\theta}=\lim _{t \rightarrow \infty} V_{\theta}\left(\sqrt{t} \operatorname{grad} U_{t}(\theta)\right)$ (here, $\left.t=n^{2}\right)$ was estimated based on Monte-Carlo simulations: 1000 Gaussian random fields were simulated under $\tilde{\theta}_{t}$; for each simulation the sample variogram $\{\hat{\gamma}(h): h \in \mathcal{H}\}$ was computed, and the first derivative of the contrast in $\tilde{\theta}_{t}$, i.e. $-\sum_{h \in \mathcal{H}} h e^{-\tilde{\theta}_{t} h}\left\{\hat{\gamma}(h)-\left(1-e^{-\tilde{\theta}_{t} h}\right)\right\}$, was calculated; the sample variance of the derivatives multiplied by $n^{2}$ gave the estimate 1.97 for $\Gamma_{\theta}$.

The term $I_{\theta}=\lim _{t \rightarrow \infty} \mathbf{H} U_{t}(\theta)$ was estimated in two ways: with the estimator $2 \pi p_{t}\left(\tilde{\theta}_{t}\right)^{2} / t$ as suggested in section 2.4 and with Monte-Carlo simulations. In the former way, the estimate of $I_{\theta}$ is 0.20 . The second way was carried out as follows: for each of the 1000 simulated Gaussian fields mentioned above, the second derivative of the contrast in $\tilde{\theta}_{t}$, i.e. $\sum_{h \in \mathcal{H}} h^{2} e^{-\tilde{\theta}_{t} h}\left[e^{-\tilde{\theta}_{t} h}-\{\hat{\gamma}(h)-(1-\right.$ $\left.\left.e^{-\tilde{\theta}_{t} h}\right)\right\}$ ], was computed; then, the sample mean of these derivatives gave the estimate 0.27 for $I_{\theta}$.

Thus, the estimate of the limit variance $\Gamma_{\theta} /\left(n I_{\theta}\right)^{2}$ of $\tilde{\theta}_{t}$ is 0.07 when $I_{\theta}$ is assessed by simulations and 0.12 when $I_{\theta}$ is computed from the CB-posterior distribution. The density function of the limit distribution $\mathcal{N}\left(\tilde{\theta}_{t}, \Gamma_{\theta} /\left(n I_{\theta}\right)^{2}\right)$ is 


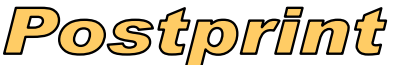

Version définitive du manuscrit publié dans / Final version of the manuscript

published in : Statistical Methodology, 2009, DOI: 10.1016/j.stamet.2009.03.003

drawn in Figure 1 (right). The continuous and dashed lines show this density when the estimate of the limit variance is 0.07 and 0.12 , respectively. The true value $\theta=1$ belongs to the $95 \%$-confidence interval whatever the estimate of the limit variance is. We see how the two versions of the limit density are different from the CB-posterior density.

To assess the efficiency of the method, the coverage rate of the $95 \%$-confidence interval was measured by applying the estimation procedure to 1000 simulated fields. The coverage rate was $94.6 \%$ when the estimate of $I_{\theta}$ is based on MonteCarlo simulations and $94.7 \%$ when the estimate of $I_{\theta}$ comes from the contrastbased posterior density.
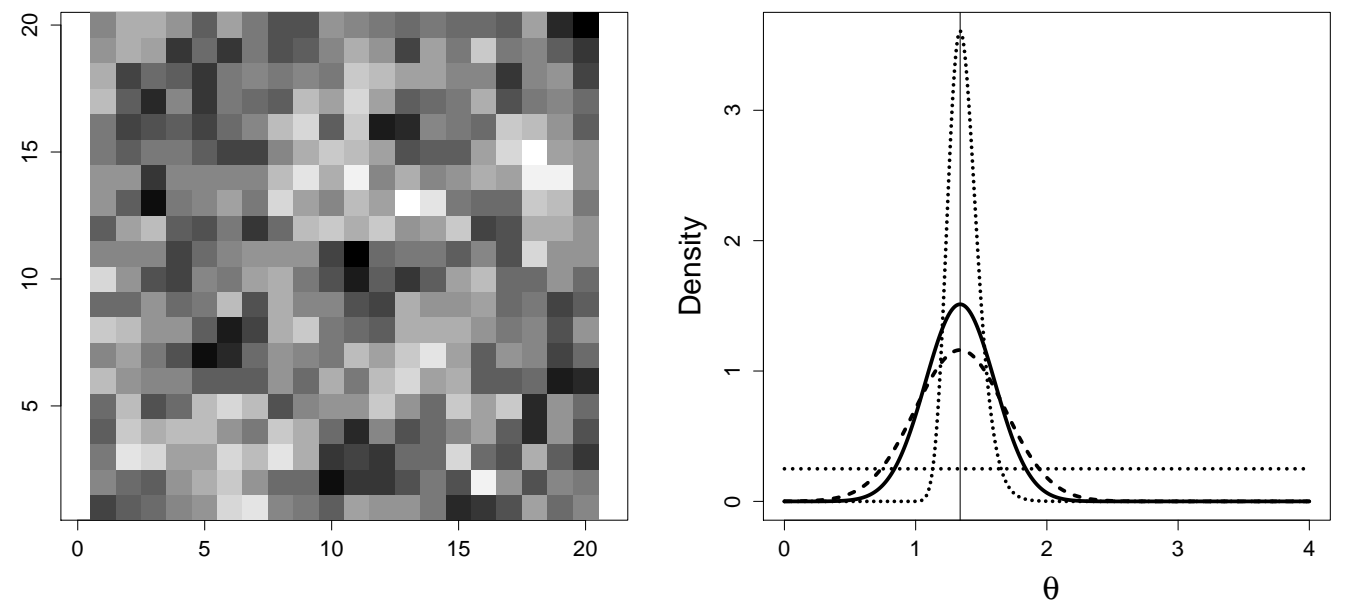

Figure 1: Left: realization of a centered Gaussian random field with exponential variogram parameterized with $\theta=1$, over a $20 \times 20$ square-grid. Right: prior density (horizontal dotted line), contrast-based posterior density (dotted curve), density function of the limit distribution $\mathcal{N}\left(\tilde{\theta}_{t}, \Gamma_{\theta} /\left(n I_{\theta}\right)^{2}\right)$ (continuous and dashed lines when the estimate of the limit variance is based on simulations and when it is based on the posterior distribution), and MAP estimator (vertical line). 


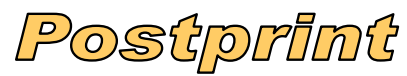

Version définitive du manuscrit publié dans / Final version of the manuscript

published in : Statistical Methodology, 2009, DOI: 10.1016/j.stamet.2009.03.003

\subsection{Pseudo-likelihood estimation of a Markovian spatial model}

This simulated case illustrates the application of the method for a bivariate parameter. Here, the CB-posterior distribution is different from the limit distribution of the estimator; it cannot be directly used to make inference but can be used for estimating $I_{\theta}$.

We built a data set by simulating a spatial Markov field $X$ with two states, 0 and 1. The model is defined by the conditional probability of $X_{i}$ given $X_{j}$, $j \in V(i)(V(i)$ is the set of the four nearest neighbors of $i$ ) satisfying (Guyon, 1985)

$$
\begin{aligned}
P_{\theta}\left(X_{i} \mid X_{j}, j \neq i\right) & =P_{\theta}\left(X_{i} \mid X_{j}, j \in V(i)\right) \\
& =\frac{\exp \left(\theta_{1} X_{i}+\theta_{2} \sum_{j \in V(i)} X_{i} X_{j}\right)}{\left\{1+\exp \left(\theta_{1}+\theta_{2} \sum_{j \in V(i)} X_{j}\right)\right\}} .
\end{aligned}
$$

The field was simulated on a $n \times n$ square grid $\mathcal{I}$ (here, $t=n^{2}=20^{2}$ ); see Figure 2 (left).

The classical likelihood cannot be analytically calculated for this model. Therefore, a pseudo-likelihood was proposed to make inference (Guyon, 1985). The pseudo-likelihood is the product of the conditional probabilities $\prod_{i \in \mathcal{I}} P_{\theta}\left(X_{i}\right.$ $\left.X_{j}, j \neq i\right)$. To estimate $\theta_{1}$ and $\theta_{2}$, we applied the estimation method proposed in this article by using a uniform prior density over $[-1.5,1.5]^{2}$ and the contrast corresponding to the pseudo-likelihood:

$$
U_{n^{2}}(\alpha)=-\frac{1}{n^{2}} \sum_{i \in \mathcal{I}} \log P_{\alpha}\left(X_{i} \mid X_{j}, j \in V(i)\right) .
$$

The CB-posterior density is shown in Figure 2 (center). The MAP estimate is $\tilde{\theta}_{t}=(-0.21,0.38)$.

To give the limit distribution $\mathcal{N}\left(\tilde{\theta}_{t}, I_{\theta}^{-1} \Gamma_{\theta} I_{\theta}^{-1} / n^{2}\right)$ of the estimator, matrices $\Gamma_{\theta}$ and $I_{\theta}$ must be estimated. We computed the gradient and the Hessian of the contrast for $N=1000$ Markov fields simulated under $\tilde{\theta}_{t}$, and we used the sample variance of the gradients for estimating $\Gamma_{\theta}$ and the sample mean of the Hessians 


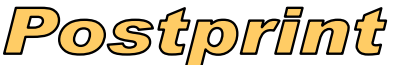

Version définitive du manuscrit publié dans / Final version of the manuscript

published in : Statistical Methodology, 2009, DOI: 10.1016/j.stamet.2009.03.003

for estimating $I_{\theta}$; see Appendix D. The estimate of the limit variance matrix $I_{\theta}^{-1} \Gamma_{\theta} I_{\theta}^{-1} / n^{2}$ was finally

$$
\left(\begin{array}{cc}
0.14 & -0.055 \\
-0.055 & 0.022
\end{array}\right) .
$$

Almost the same limit variance matrix was obtained when $I_{\theta}$ was estimated by fitting a normal density to the CB-posterior density as suggested in section 2.4. Figure 2 (right) shows the limit density function of the estimator together with the 95\%-confidence zone. We can see that the true parameter belongs to this zone. Moreover, Figure 2 shows the limit density is quite close from the posterior density. The pseudo-likelihood which takes account of short-distance interactions certainly brings almost the same information than the likelihood brings. It has however to be noted that this would not be the case if long-distance interactions had been introduced in the spatial Markov model.
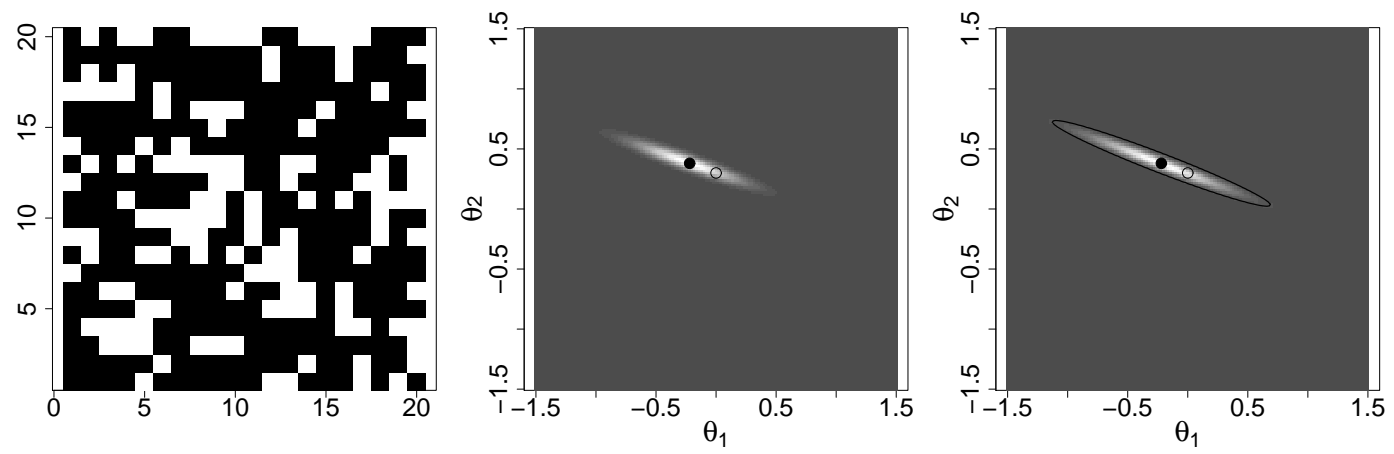

Figure 2: Left: realization of a Markovian spatial process with two states over a $20 \times 20$ grid. Center: contrast-based posterior density. Right: limit density $\mathcal{N}\left(\tilde{\theta}_{t}, I_{\theta}^{-1} \Gamma_{\theta} I_{\theta}^{-1} / n^{2}\right)$. On the center and right panels, the MAP estimate and the true parameter are drawn with a black dot and a circle, respectively. On the right panel, the continuous line circumscribes the 95\%-confidence zone.

\section{Application in spatial statistics: real data}

This section presents a case where the CB-posterior distribution can be directly used to make inference in the frequentist and Bayesian ways (the estimation 
of the limit matrices $I_{\theta}$ and $\Gamma_{\theta}$ being not required). The method is applied to a real data set dealing with soil roughness.

\subsection{Cylinder-based autosimilar model}

Soil roughness plays an important role in rain water absorption, pond and streaming. It also modifies reflectance properties of soils used to estimate soil moisture with remote detection for example. An experiment was carried out to measure soil roughness at a small scale. Soil heights were measured every $2 \mathrm{~mm}$ along $1.18 \mathrm{~m}$-transects in a cultivated field (Bertuzzi et al., 1995). Figure 3 (top) shows the distributions of heights for two among twelve sampled transects. The mean height computed from the 12 transects is $7.6 \mathrm{~mm}$, the maximum is $22.9 \mathrm{~mm}$.

Object based models (Lantuéjoul, 2002, chap. 14) have been used to describe soil surface (Bertuzzi et al., 1995; Goulard and Chadœuf, 1994). Here, we use a cylinder-based autosimilar models defined as follows. For any $x \in \mathbb{R}^{2}$ and $r>0$, let $f(x, r)=r 1_{\{\|x\|<r\}}$ be the function describing the cylinder which is centered in $x$ and whose radius and height are equal to $r\left(1_{\{\cdot\}}\right.$ is the indicator function). In addition, let $(X, R)$ be a marked Poisson point process defined over $\mathbb{R}^{2} \times \mathbb{R}_{+}^{*}$ with intensity function $\mu(x, r)=\alpha \exp \{-\beta r\}$. The random surface $Y$ representing the soil surface is defined by

$$
Y_{M}=\sum_{(x, r) \in(X, R)} f(x-M, r)
$$

\subsection{Parameter estimation using moments}

For such a process, it is difficult to calculate the joint distribution of the heights whereas the moments can easily be written. We propose to estimate the bivariate parameter vector $\theta=(\alpha, \beta)$ using the first two moments: $\hat{\mu}_{A}=$ $\left(\frac{1}{\nu(A)} \int_{A} Y_{M} d M, \frac{1}{\nu(A)} \int_{A} Y_{M}^{2} d M\right)$, where $A$ is the set of the sampled transects and $\nu(A)$ is its measure.

If border effects are neglected, the expected value of $\hat{\mu}_{A}$ is

$$
E\left(\hat{\mu}_{A}\right)=\left(6 \pi \frac{\alpha}{\beta^{4}}, 36 \pi^{2} \frac{\alpha^{2}}{\beta^{8}}+24 \pi \frac{\alpha}{\beta^{5}}\right) .
$$




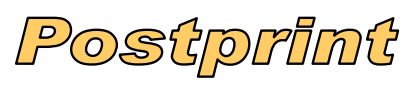

Version définitive du manuscrit publié dans / Final version of the manuscript

published in : Statistical Methodology, 2009, DOI: 10.1016/j.stamet.2009.03.003

Moreover, the variance matrix of $\hat{\mu}_{A}$ satisfies

$$
\nu(A) \operatorname{var}\left(\hat{\mu}_{A}\right) \rightarrow V
$$

where the components of $V$ are

$$
\begin{aligned}
& V_{11}=5 ! \frac{16}{3} \frac{\alpha}{\beta^{6}} \\
& V_{12}=6 ! \frac{16}{3} \frac{\alpha}{\beta^{7}}+(5 !) 64 \pi \frac{\alpha^{2}}{\beta^{10}} \\
& V_{22}=7 ! \frac{16}{3} \frac{\alpha}{\beta^{8}}+\{(6 !) 128 \pi+(10 !) 32 \kappa\} \frac{\alpha^{2}}{\beta^{11}}+(3 !)(5 !) 128 \pi^{2} \frac{\alpha^{3}}{\beta^{14}},
\end{aligned}
$$

with $\kappa=\int_{0}^{1} \int_{0}^{1}\left(\arccos (u)-u \sqrt{1-u^{2}}\right)\left(\arccos (v)-v \sqrt{1-v^{2}}\right) \frac{(u v)^{5}}{(u+v)^{11}} d u d v$.

The estimation method is applied by using a uniform prior over $[1,100] \times[1,5]$ and a contrast based on the weighted least squares of the first two moments:

$$
U_{A}(\theta)=\left(\hat{\mu}_{A}-E\left(\hat{\mu}_{A}\right)\right)^{\prime} V^{-1}\left(\hat{\mu}_{A}-E\left(\hat{\mu}_{A}\right)\right) / 2 .
$$

For this contrast, the matrices $I_{\theta}$ and $\Gamma_{\theta}$ are equal and their component $(i, j)$ is

$$
\frac{\partial E\left(\hat{\mu}_{A}\right)^{\prime}}{\partial \theta_{i}} V^{-1} \frac{\partial E\left(\hat{\mu}_{A}\right)}{\partial \theta_{j}} \text {. }
$$

Consequently, $I_{\theta}^{-1} \Gamma_{\theta} I_{\theta}^{-1}=I_{\theta}^{-1}$ and the $\mathrm{CB}$-posterior density can be used as an approximation of the limit density of the MAP estimator $\tilde{\theta}_{A}$ or as a posterior distribution of the parameter $\theta$ (see section 2.4). Figure 3 (bottom) shows the joint CB-posterior distribution and the marginals. The MAP estimate of $\theta$ is $\tilde{\theta}_{A}=(46.6,3.28)$. Marginal 95\%-confidence intervals of $\alpha$ and $\beta$ are $[36.1,58.5]$ and $[3.07,3.48]$, respectively.

\section{Discussion}

\subsection{Summary}

We have proposed an estimation method exploiting a contrast-based posterior distribution (CBPD). This method includes the classical likelihood-based procedures (MLE and Bayesian estimation), but has been mainly developed to circumvent difficulties encountered with the likelihood by generalizing the Bayesian 


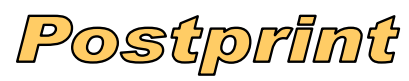

Version définitive du manuscrit publié dans / Final version of the manuscript

published in : Statistical Methodology, 2009, DOI: 10.1016/j.stamet.2009.03.003
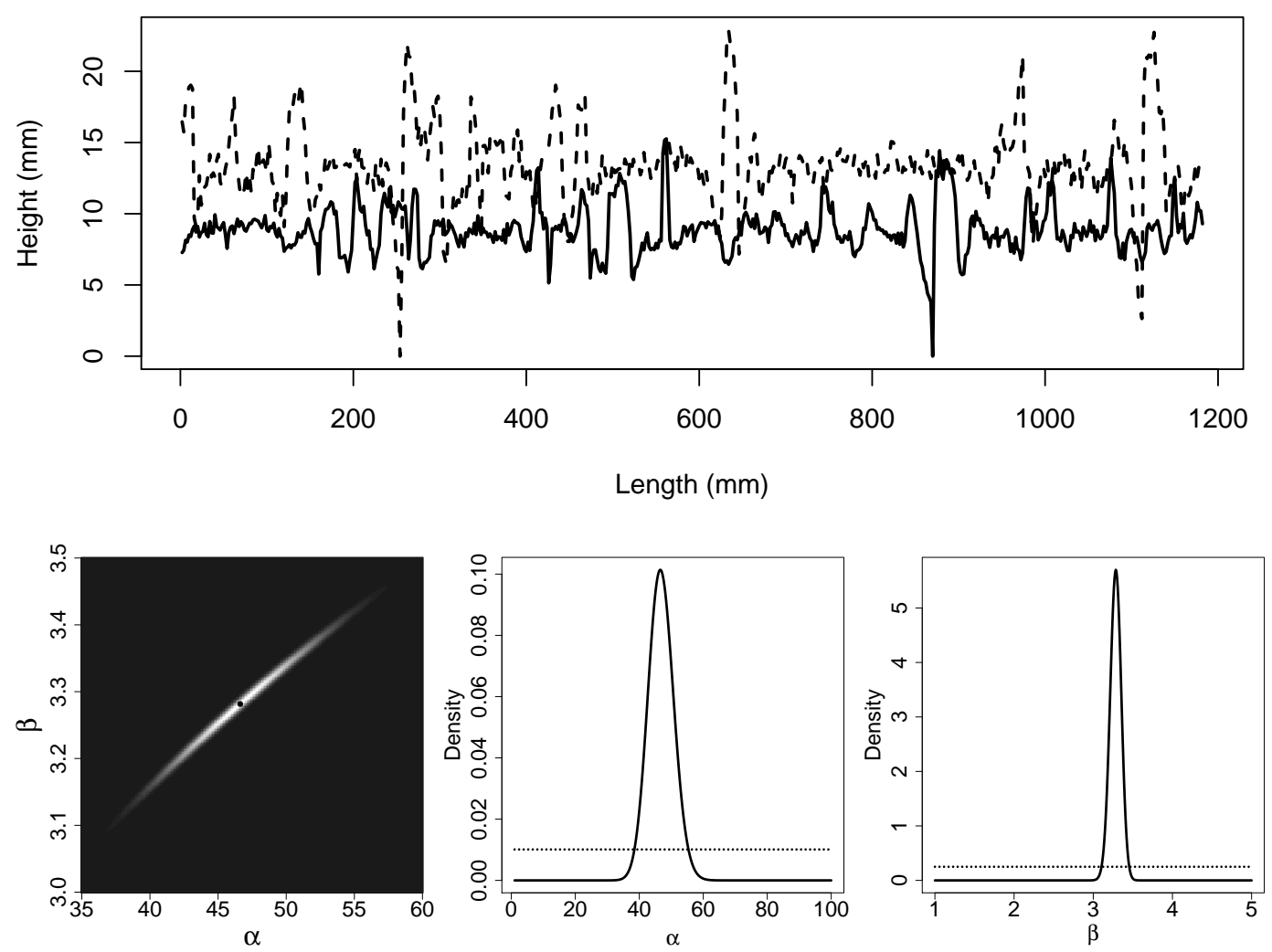

Figure 3: Top: distribution of heights for two transects. Bottom left: contrastbased posterior density for $(\alpha, \beta)$; the MAP estimate is at the black dot. Bottom center and right: contrast-based posterior marginal densities for $\alpha$ et $\beta$ (continuous lines) and prior marginal densities (dashed lines).

formula of the posterior distribution, so extending the proposal of Lin (2006). The CBPD can be used to make frequentist inference and, in specific situations, Bayesian inference. In case of frequentist inference, the use of the CBPD allows the reduction of analytical calculations usually required to compute the limit variance matrix of the estimator. In this article, the method has been applied to spatial data sets, but can be applied to other cases where likelihood-based procedures are not appropriate. 


\subsection{Frequentist and Bayesian inference}

In the frequentist viewpoint, the CBPD can be used to provide a point estimator (the posterior mode) and the limit distribution of this estimator. The limit distribution is directly approximated by the CBPD if the variance of the gradient vector of the contrast is equal to the inverse of the limit Hessian matrix of the contrast (i.e. $I_{\theta}^{-1} \Gamma_{\theta} I_{\theta}^{-1}=I_{\theta}^{-1}$; see the third application). In this case, it is not required to calculate and estimate the variance matrix of the estimator. In other cases, the limit distribution is not directly available, but the Hessian matrix of the contrast can be easily estimated from the CBPD and, consequently, the calculation of the second derivatives of the contrast is avoided (see the first two applications). It has to be noted that using Bayesian calculation to make frequentist estimation has been proposed in the literature (Robert and Hwang, 1996; Robert and Titterington, 1998; Jacquier et al., 2007), but the proposals were restricted to maximum likelihood estimation.

In the Bayesian viewpoint, the CBPD can be used as a classical posterior distribution when $I_{\theta}^{-1} \Gamma_{\theta} I_{\theta}^{-1}=I_{\theta}^{-1}$, as in the third application. However, the CBPD does not coincide with the classical posterior distribution: It has to be viewed as a posterior distribution based on the information brought by the contrast which is used.

Regarding the reconciliation of the frequentist and the Bayesian approaches, it has to be noted that the Bernstein-Von Mises (BVM) theorem, translated to our context, does not hold in general. The classical BVM theorem asserts that, under specific conditions, the posterior distribution converges to a Gaussian distribution with variance matrix equal to the asymptotic variance of the maximum likelihood estimator (Freedman, 1999). In this paper we saw that the CBPD is asymptotically equivalent to a Gaussian distribution with variance matrix $I_{\theta}^{-1}$ whereas the asymptotic variance matrix of the minimum contrast estimator is $I_{\theta}^{-1} \Gamma_{\theta} I_{\theta}^{-1}$. 


\subsection{Limits of the approach and perspectives}

Even if the proposed procedure has advantages, it also faces two classical limits: the choice of the prior distribution (or the penalization function in the frequentist viewpoint) which can influence the posterior inference, and the choice of the contrast. Regarding the former limit, we refer to Clarke and Gustafson (1998) and Rootzén and Olsson (2006) for example. Regarding the choice of the contrast, two comments arise.

The first comment concerns the possibility to build a contrast such that $I_{\theta}^{-1} \Gamma_{\theta} I_{\theta}^{-1}=I_{\theta}^{-1}$ (case where our method has favorable properties). Necessary and sufficient conditions for obtaining this equation are not known to us. However, sufficient conditions can be given. For example, using a coding technique (Besag, 1975) based on conditional independence between sets of data is a sufficient condition. We could have applied such a technique instead of the pseudo-likelihood method for the estimation of the parameters of the Markovian spatial model (second simulated case-study). But, the use of the coding method would have induced a loss of information (Guyon, 1985). It is also possible to obtain $I_{\theta}^{-1} \Gamma_{\theta} I_{\theta}^{-1}=I_{\theta}^{-1}$ for specific models by rescaling the contrast with a variance matrix. We applied this approach in the real case-study because we could provide the analytical form for the variance matrix of the sample moments. To apply the same approach for the estimation of the range parameter of a variogram (first case-study), we should have modeled the variance of the sample variogram. However, such a practice is not common in geostatistics when the field is not assumed to be Gaussian (and it may be cumbersome). It has to be noted that if an approximate likelihood is used and its discrepancy from the true likelihood can be analytically quantified, it may be possible to assess the discrepancy between $I_{\theta}^{-1} \Gamma_{\theta} I_{\theta}^{-1}$ and $I_{\theta}^{-1}$, and to correct the CBPD accordingly.

Our second comment concerns the information brought by contrasts. We see that in the real case-study the two estimators are strongly correlated. We could have tried to use another contrast to avoid correlation. For example, together with the sample mean, we could have used the covariance at a given distance instead of the variance to get two moments which are less correlated. However, the calculation of the expected value and the variance-covariance of these moments 


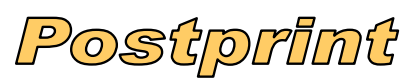

Version définitive du manuscrit publié dans / Final version of the manuscript

published in : Statistical Methodology, 2009, DOI: 10.1016/j.stamet.2009.03.003

is much more tricky. Thus, to be able to derive analytical expressions and apply the method as it is presented, the choice of the contrast is limited. Nevertheless, simulations could be used to circumvent this difficulty. This could be an interesting extension of the estimation method proposed in this paper.

\section{References}

Beaumont, M. A., W. Zhang, and D. J. Balding (2002). Approximate bayesian computation in population genetics. Genetics 162, 2025-2035.

Bertuzzi, P., L. Garcia-Sanchez, J. Chadøuf, J. Guérif, M. Goulard, and P. Monestiez (1995). Modelling surface roughness by a boolean approach. European Journal of Soil Science 46, 215-220.

Besag, J. (1975). Statistical analysis of non-lattice data. The Statictician 24, 179-195.

Caillot, P. and F. Martin (1972). Le modèle bayésien. Annales de l'IHP, section B 8, 183-210.

Chilès, J.-P. and P. Delfiner (1999). Geostatistics. Modeling Spatial Uncertainty. New York: Wiley.

Clarke, B. and P. Gustafson (1998). On the overall sensitivity of the posterior distribution to its inputs. Journal of Statistical Planning and Inference 71, $137-150$.

Dacunha-Castelle, D. and M. Duflo (1982). Probabilités et Statistiques. Problèmes ä̈ $\ddot{z} \frac{1}{2}$ Temps Mobile, Volume 2. Paris: Masson.

Diggle, P. J., J. A. Tawn, and R. A. Moyeed (1998). Model-based geostatistics. Applied Statistics 47, 299-350.

Freedman, D. (1999). On the Bernstein-Von Mises theorem with infinitedimensional parameters. The Annals of Statistics 27, 1119-1140. 


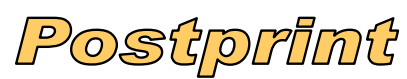

Version définitive du manuscrit publié dans / Final version of the manuscript

published in : Statistical Methodology, 2009, DOI: 10.1016/j.stamet.2009.03.003

Goulard, M. and P. Chadœuf, Bertuzzi (1994). Random boolean functions: nonparametric estimation of the intensity. application to soil surface roughness. Statistics 25, 123-136.

Guyon, X. (1985). Estimation d'un champ par pseudo-vraisemblance conditionnelle : étude asymptotique et application au cas markovien. In E. des Facultés Universitaires de St Louis (Ed.), Actes de la 6ème rencontre Franco-Belge de Statitisticiens.

Jacquier, E., M. Johannes, and N. Polson (2007). Mcmc maximum likelihood for latent state models. Journal of Econometrics 137, 615-640.

Lantuéjoul, C. (2002). Geostatistical Simulation, Models and Algorithms. Berlin: Springer.

Lin, L. (2006). Quasi bayesian likelihood. Statistical Methodology 3, 444-455.

Markatou, M. (2000). Mixture models, robustness, and the weighted likelihood methodology. Biometrics 56, 483-486.

McCullagh, P. and J. A. Nelder (1989). Generalized Linear Models, 2nd Ed. London: Chapman \& Hall.

Molchanov, I. (1997). Statistics of the Boolean Model for Practitioners and Mathematicians. Chichester: Wiley.

Møller, J. (Ed.) (2003). Spatial Statistics and Computational Methods, Volume 173 of Lecture Notes in Statistics. New York: Springer-Verlag.

Robert, C. P. and G. Casella (1999). Monte Carlo Statistical Methods. New York: Springer.

Robert, C. P. and J. T. G. Hwang (1996). Maximum likelihood estimation under order restrictions by the prior feedback method. Journal of the American Statistical Association 91, 167-172. 


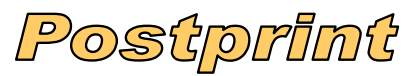

Version définitive du manuscrit publié dans / Final version of the manuscript

published in : Statistical Methodology, 2009, DOI: 10.1016/j.stamet.2009.03.003

Robert, C. P. and D. M. Titterington (1998). Reparameterization strategies for hidden Markov models and Bayesian approaches to maximum likelihood estimation. Statistics and Computing 8, 145-158.

Rootzén, H. and J. Olsson (2006). On the influence of the prior distribution in image reconstruction. Computational Statistics 21, 431-444.

Serfling, R. J. (2002). Approximation Theorems of Mathematical Statistics. Wiley.

Stein, M. L. (1999). Interpolation of Spatial Data: Some Theory for Kriging. New York: Springer-Verlag.

Van Lieshout, M. N. M. and E. W. Van Zwet (2001). Exact sampling from conditional boolean models with applications to maximum likelihood inference. Advances in Applied Probability 33, 339-353.

Walker, S. and N. L. Hjort (2001). On Bayesian consistency. Journal of the Royal Statistical Society B 63, 811-821.

Wei, G. C. G. and M. A. Tanner (1990). A monte carlo implementation of the em algorithm and the poor man's data augmentation algorithms. Journal of the American Statistical Association 85, 699-704.

Zhang, H. (2002). On estimation and prediction for spatial generalized linear mixed models. Biometrics 58, 129-136.

\section{A Hypotheses for minimum contrast estimation}

$\mathrm{H}_{1}: \Theta \subset \mathbb{R}^{p}, p<\infty$, is compact and $\theta$ is in the interior of $\Theta$,

$\mathrm{H}_{2}: \alpha \mapsto K(\alpha, \theta)$ has a strict minimum at $\theta$,

$\mathrm{H}_{3}: \alpha \mapsto U_{t}(\alpha)$ is $C^{2}$ (it has two continuous derivatives) over $\Theta$,

$\mathrm{H}_{4}$ : the normalized gradient vector $\sqrt{t} \operatorname{grad} U_{t}(\theta)$ (first derivatives of $U_{t}(\theta)$ with respect to $\theta$ ) converges in law to the normal distribution $\mathcal{N}\left(0, \Gamma_{\theta}\right)$ :

$$
\sqrt{t} \operatorname{grad} U_{t}(\theta) \rightarrow \mathcal{N}\left(0, \Gamma_{\theta}\right) \quad \text { in law as } t \rightarrow \infty
$$




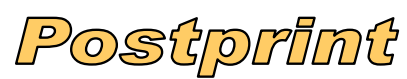

Version définitive du manuscrit publié dans / Final version of the manuscript

published in : Statistical Methodology, 2009, DOI: 10.1016/j.stamet.2009.03.003

$\mathrm{H}_{5}$ : the Hessian matrix $\mathbf{H} U_{t}(\theta)$ (second derivatives of $U_{t}(\theta)$ with respect to $\theta$ ) converges in probability to an invertible matrix $I_{\theta}$ :

$$
\mathbf{H} U_{t}(\theta) \rightarrow I_{\theta} \quad \text { in probability as } t \rightarrow \infty
$$

$\mathrm{H}_{6}: \sup _{k l \mid \epsilon \epsilon}\left|\mathbf{H}_{k l} U_{t}(\theta+\beta)-\mathbf{H}_{k l} U_{t}(\theta)\right| \rightarrow 0$ in probability, where $\epsilon>0$ and $\mathbf{H}_{k l}$ is $\|\beta\|<\epsilon$

the component $(k, l), 1 \leq k, l \leq p$, of the Hessian operator.

Under these assumptions, the minimum contrast estimator is consistent and asymptotically normal (Dacunha-Castelle and Duflo, 1982): as $t \rightarrow \infty$,

- $\hat{\theta}_{t}$ converges in probability to $\theta$ and

- $\sqrt{t}\left(\hat{\theta}_{t}-\theta\right)$ converges in law to the Gaussian distribution $\mathcal{N}\left(0, I_{\theta}^{-1} \Gamma_{\theta} I_{\theta}^{-1}\right)$.

\section{B Proof of equation (2)}

Since $\tilde{\theta}_{t}$ satisfies $\operatorname{grad} p_{t}\left(\tilde{\theta}_{t}\right)=0$,

$$
0=-t c\left(\tilde{\theta}_{t}\right) \operatorname{grad} U_{t}\left(\tilde{\theta}_{t}\right)+\operatorname{grad} c\left(\tilde{\theta}_{t}\right)
$$

Then, applying a first order Taylor's expansion for $\operatorname{grad} U_{t}\left(\tilde{\theta}_{t}\right)$ around $\hat{\theta}_{t}$ yields

$$
0=-t c\left(\tilde{\theta}_{t}\right)\left\{\operatorname{grad} U_{t}\left(\hat{\theta}_{t}\right)+\left(\mathbf{H} U_{t}\left(\hat{\theta}_{t}\right)\right)\left(\tilde{\theta}_{t}-\hat{\theta}_{t}\right)\right\}\left(1+o_{\text {proba }}(1)\right)+\operatorname{grad} c\left(\tilde{\theta}_{t}\right) .
$$

In this equation, $\operatorname{grad} U_{t}\left(\hat{\theta}_{t}\right)=0$ because $\hat{\theta}_{t}$ is the maximizer of $U_{t}(\cdot)$. Moreover, applying zero order Taylor's expansions for $c\left(\tilde{\theta}_{t}\right), \mathbf{H} U_{t}\left(\hat{\theta}_{t}\right)$ and $\operatorname{grad} c\left(\tilde{\theta}_{t}\right)$ around $\theta$ yields

$$
\begin{aligned}
0 & =-t c(\theta)\left(\mathbf{H} U_{t}(\theta)\right)\left(\tilde{\theta}_{t}-\hat{\theta}_{t}\right)\left(1+o_{\text {proba }}(1)\right)+\operatorname{grad} c(\theta) \\
& =-t c(\theta) I_{\theta}\left(\tilde{\theta}_{t}-\hat{\theta}_{t}\right)\left(1+o_{\text {proba }}(1)\right)+\operatorname{grad} c(\theta)
\end{aligned}
$$

since $\lim _{t \rightarrow \infty} \mathbf{H} U_{t}(\theta)=I_{\theta}$ in probability. Then equation (2) follows. 


\section{Proof of equation (3)}

Let $\delta>0$. For any a such that $\sup _{1 \leq i \leq p}\left|a_{i}\right|<t^{\delta}$, a third order Taylor's expansion yields

$\log p_{t}\left(\tilde{\theta}_{t}+a / \sqrt{t}\right)-\log p_{t}\left(\tilde{\theta}_{t}\right)=-\sqrt{t} a^{\prime} \operatorname{grad} U_{t}\left(\tilde{\theta}_{t}\right)-\frac{1}{2} a^{\prime} I_{\theta} a+o_{\text {proba }}\left(t^{2 \delta}+t^{3 \delta-1 / 2}\right)$.

Given that $\operatorname{grad} U_{t}\left(\hat{\theta}_{t}\right)=0$ (definition of the classical minimum contrast estimator $\left.\hat{\theta}_{t}\right)$ and that $\tilde{\theta}_{t}-\hat{\theta}_{t}=o_{\text {proba }}\left(t^{-1+\delta}\right) \mathbf{1}_{p}$ where $\mathbf{1}_{p}$ is the unit vector of size $p$, the dimension of $\Theta$ (see eq. (2)), the previous equation becomes

$$
\log p_{t}\left(\tilde{\theta}_{t}+a / \sqrt{t}\right)-\log p_{t}\left(\tilde{\theta}_{t}\right)=-\frac{1}{2} a^{\prime} I_{\theta} a+o_{\text {proba }}\left(t^{2 \delta}+t^{3 \delta-1 / 2}\right) .
$$

Ensuring that $\delta<1 / 2$ (and not only $\delta>0$ ), then

$$
\begin{aligned}
\log p_{t}\left(\tilde{\theta}_{t}+a / \sqrt{t}\right)-\log p_{t}\left(\tilde{\theta}_{t}\right) & =-\frac{1}{2} a^{\prime} I_{\theta} a+o_{\text {proba }}\left(t^{2 \delta}\right) \\
& =-\frac{1}{2} a^{\prime} I_{\theta} a\left\{1+o_{\text {proba }}(1)\right\} .
\end{aligned}
$$

Let us introduce $g_{t}: a \mapsto t^{-p / 2} p_{t}\left(\tilde{\theta}_{t}+a / \sqrt{t}\right)$ defined over $\mathbb{R}^{p}$. This density function satisfies, from the previous result,

$$
g_{t}(a) \underset{t \rightarrow \infty}{\sim} t^{-p / 2} p_{t}\left(\tilde{\theta}_{t}\right) \exp \left(-\frac{1}{2} a^{\prime} I_{\theta} a\right) .
$$

Since $g_{t}(\cdot)$ is a density function and given the form of the right-hand-side term of this equation, $g_{t}(\cdot)$ is equivalent to the density function of the normal law with variance matrix $I_{\theta}^{-1}$. Equation (3) is then obtained with the change of variable $\alpha=\tilde{\theta}_{t}+a / \sqrt{t}$.

\section{Spatial Markovian model: Limit matrices}

$I_{\theta}$ and $\Gamma_{\theta}$ satisfy $I_{\theta}=\operatorname{var}\left(Z_{0}\right)$ and $\Gamma_{\theta}=M_{0}+4 \sum_{0 \leq i_{1}, i_{2} \leq 2} M_{i}$ where $M_{i}=$ $\operatorname{cov}\left(Z_{0}, Z_{i}\right), i \in \mathcal{I}$, and

$$
Z_{i}=\left(X_{i}-\frac{\exp \left(\theta_{1}+\theta_{2} \sum_{j \in V(i)} X_{j}\right)}{1+\exp \left(\theta_{1}+\theta_{2} \sum_{j \in V(i)} X_{j}\right)}\right)\left(\begin{array}{c}
1 \\
\sum_{j \in V(i)} X_{j}
\end{array}\right) .
$$

